\title{
Skin Color in Stillbirth Disparities: The Social Work Application of Evidence-based Practice via Information Triad
}

\author{
Ronald Hall \\ School of Social Work, Michigan State University, USA
}

\begin{abstract}
By medical explanation, a stillbirth pertains to the birth of an unborn child where indications of life are lacking. Stillbirths do not occur uniformly among pregnant women but by disparity where black women are at greatest risk as documented by the EBP information triad. As per EBP stillbirth research, experts and client input is critical. The results of reference to such information suggest that having dark skin is motivation to apply bleaching creams, which contain toxic substances. By EBP methods it is here suggested that by way of the placenta toxins enter the fetus of black mothers resulting in stillbirth disparity. In the EBP meticulous collection of data, expert consultation and client input the resolution of stillbirth disparities will be less subject to inferior problem-solving strategies. Skin color in the study of stillbirth disparities can greatly reduce events among dark-skinned mothers if practitioners advise pregnant black women to cease bleaching until such time that their child is born. By eliminating race from the dynamics of scientific investigation of skin color per EBP Social Work will be served in meeting its challenges well into the $21^{\text {st }}$ century and beyond.
\end{abstract}

Keywords Stillbirths, Skin Color, Race, Evidence-based Practice

\section{Introduction}

Defined by medical explanation a stillbirth pertains to the birth of an unborn child where indications of life are lacking. Subsequently the fetus emerges as deceased following exit from the mother's birth canal. By race, as proxy for skin color, stillbirths do not occur uniformly among pregnant women but occur by racial disparity as documented in information evidence. Aside from documented racial demographics, there is an assortment of factors that may precipitate a stillbirth event including diabetes, hypertension, renal dysfunction and other abnormalities (Soh, Nelson-Piercy, Dib, Westgren \& McCowan, et al., 2015). Such factors apply regardless of racial or ethnic demographic categories. However, stillbirths among Caucasian race babies i.e.: light-skinned occurs at a much lower rate than that among Negroid race babies i.e.: dark-skinned (Tyler, Grady, Grigorescu, Luke \& Todem, 2012). This is so despite controlling for socio-economic and other demographic factors. Given to a Eurocentric perspective, which dominates the Social Work academy in its dedication to the demographic category of race, is omission of the most critical factor associated with stillbirth events heretofore suggested as dark skin (Murdoch, 2009).

According to Ore (2011) the definition of race relative to dark skin pertains to a community of persons who perceive themselves and are perceived by others to carry a distinct assortment of biological characteristics. Said characteristics frequently exist in the form of visible physiological traits most dramatic in the observation of dark skin. This definition is discrete from ethnicity, which encompasses language, religion, customs, and food choices, etc. Conversely, race and ethnicity are commonly applied interchangeably but in fact relative to stillbirth events are two distinct categories.

Among anthropologists, Garn and Coon (2009) divide race into three classifications consisting of Negroid, Caucasoid and Mongoloid. The origins of Negroid extend from Africa; Caucasoid from Europe; and Mongoloid from Asia. Each group is discrete in its visual appearance whereby in skin color Negroid is darkest, Caucasoid is lightest and Mongoloid is a medium version of the two polar extremes (Glasker, 2014). Therefore, Mongoloid is dark compared to Caucasoid but light compared to Negroid.

While race is widely applied as a demographic category, its definition relative to the three classifications is inconsistent. This inconsistency may vary across cultures, across religions and across numerous national boundaries. Race may also vary across spans of time and across various 
academic disciplines. Those members of the academy who presume to formulate a universal definition of race are too often motivated by personal agendas and the institutional objectives of their intellectual preferences $(\mathrm{Hu}, 2008)$. Subsequently race has no statistically unquestioned applicable method of measurement. Those statistical measures, which do exist, are limited to nominal data given the inconsistent subjective nature of the definition. Therefore, as nominal data any statistical calculations considering race have no mathematical significance which the assessment of stillbirth disparities makes readily apparent (Dubriwny, Bates \& Bevan, 2004).

Skin color similar to race is also widely applied as a demographic category in its proxy associations with race. However, its application is more on an operational basis as a clue to race and unlike race its definition is consistent. Any expressed definition of skin color is universally bound to the melanin content in human skin. Therefore, references to the definition of skin color at root, does not vary across cultures, across religions or across national boundaries. It is biologically consistent across spans of time and across a variation of academic disciplines. Those few members of the academy who reference skin color are not limited by personal agendas or institutional objectives. What's more, skin color is not limited to nominal data measures but is amenable to all statistical data categories including the ordinal, interval and ratio. This allows for prudent scientific investigation and objective statistical measurements (Krause, 2012).

The universal consistency of skin color is a product of melanin. Melanin is a biological fact of all human populations $(\mathrm{Hu}, 2008)$. Given to an end product of metabolism relative to amino acid tyrosine, the associated melanin is also found in skin moles and dark dermal melanocytes which are pigment cells. They are characteristic of most dark-skinned human populations. Melanin may also exist as diffuse brown spots located in the skin's epidermis. When melanin occurs in the highest concentration the darkest of complexions is then visually apparent (King, Yerger, Whembolua, Bendel \& Kittles, et. al., 2009). While the definition of skin color is universally consistent, how it is valued and perceived by the assortment of populations around the world may be inconsistent and thus subject to unspoken inconsistency motives.

The objective of this paper using an evidence-based approach is to assess skin color disparities in stillbirth events via dark skin and thus enable Social Work practitioners to better serve their clients confronted with health pathologies. To accomplish this objective will be accrued in the following text: (a) stillbirth research: empirical evidence; (b) stillbirth experts: medical authorities; (c) stillbirth clients: a blog documentation of dark skin; and (d) the application of evidence-based practice: an introduction.

\section{Stillbirth Research: Empirical Evidence}

The Centers for Disease Control (CDC) maintains detailed empirical evidence of stillbirth disparities relative to skin color implied by race. According to 2015 data, the variation in stillbirths by race is dramatic as pertains to the racial category of the mother. For Caucasian, i.e.: white mothers who have light skin the rate was 4.88 for every 1,000 live and stillbirths. Relative to Asian/Pacific Islander Mongoloid mothers who have a similar light skin stillbirth events were approximately the same at 4.68 for every 1,000 live and stillbirths. For dark-skinned, Negroid i.e.: black stillbirth rates by comparison were more than twice the rate compared to white mothers. While the rates for white mothers were 4.88 , the rate for black mothers characterized by dark skin was 10.53 stillbirth events for every 1,000 live births (CDC, 2015).

According to the World Health Organization (WHO) a stillbirth is defined as the loss of a fetus during pregnancy in excess of 20 weeks gestation (Gibbs, 2005). Several of the main causes include intrauterine growth restriction, malformations, infection, and "unexplained" abruption. Risk factors for stillbirth of the fetus includes advanced maternal age, despite accounting for medical conditions, obesity, despite controlling for gestational diabetes and hypertension, thrombophilia where odds ratios range from 1.8 to 12 , infection including "immunologic exposure," such as parvovirus B19, toxoplasmosis, and listeriosis. Heading this list of risks is dark skin via Negroid racial category. Such mothers are twice as likely to experience a stillbirth event compared to mothers of other races. Subsequently the risk for stillbirths among dark-skinned mothers doubles regardless of the availability of adequate prenatal care and state of the art reproductive technology (Gibbs, 2005).

According to the International Stillbirth Alliance (2009) the number of stillbirths per 1000 births in African (dark-skinned) countries by double digit selected far exceeds that of European (light-skinned) countries (see Table 1 and Table 2).

Table 1. African Countries by Double-Digit Select Stillbirths per 1000 Total Births

\begin{tabular}{|l|l|l|l|l|l|l|l|l|l|}
\hline 11.3 & 27.7 & 28.6 & 21.2 & 23.7 & 20.6 & 28.4 & 21.9 & 23.9 & 24.8 \\
\hline 25.1 & 25.6 & 27.4 & 25.6 & 29.6 & 23.7 & 15.1 & 33.8 & 18.0 & 25.5 \\
\hline 24.3 & 24.2 & 33.9 & 17.3 & 21.8 & 23.2 & 22.9 & 30.0 & 25.6 & 20.0 \\
\hline 16.1 & 29.2 & 13.1 & 25.9 & 25.3 & 27.4 & 41.7 & 30.1 & 25.0 & \\
\hline 26.2 & 27.1 & 16.7 & 22.0 & 26.9 & 19.5 & 22.8 & 20.4 & 10.1 & \\
\hline
\end{tabular}

International Stillbirth Alliance (2009) 
Table 2. European Countries by Double-Digit Select Stillbirths per 1000 Total Births

\begin{tabular}{|l|l|l|l|}
\hline 14.5 & 12.2 & 16.7 & 11.2 \\
\hline
\end{tabular}

International Stillbirth Alliance (2009)

In reference to the International Stillbirth Alliance (2009), those African countries where stillbirths reached double-digits select per every 1000 total births the mean was 23.96. The African country with the highest number of double-digit select stillbirths per 1000 total births was Nigeria (41.7). The African country with the lowest number of double-digit select stillbirths per 1000 total births was Tunisia (10.1). Of note, the people of Nigeria given their Negroid heritage are more often darker-skinned than those of Tunisia by mixed-race Arab Mongoloid heritage being lighter-skinned.

According to the International Stillbirth Alliance (2009) for those European countries where stillbirths reached double-digits select per every 1000 total births the mean was 13.65. The European country with the highest number of double-digits select stillbirths per 1000 total births was Georgia (16.7). The European country with the lowest number of double-digits select stillbirths per 1000 total births was Turkey (11.2). Subsequently by documented available research, dark skin implied by race is a significant associated factor in stillbirth events.

\section{Stillbirth Experts: Medical Authorities}

Hogue and Silver (2011) as experts reported evidence of stillbirth events similar to that of available research. They refer to long-standing racial disparities, which are dramatic via black/white racial comparisons. Using 2005 data experts contend that stillbirths ("fetal deaths $\geq 20$ weeks' gestation per 1000 fetal deaths and live births") events for racially dark-skinned mothers were 11.13 compared to 4.79 for racially light-skinned mothers. Considering American Indian/Alaska Natives and Hispanics who have a Mongoloid skin tone the rate of stillbirths was similarly intermediate at 6.17 and 5.44. The aforementioned racial disparities were evident for both early gestation at 28 weeks and later stillbirth events (Hogue \& Silver, 2011).

Data for stillbirth events relative to race as skin color in Europe is similar to that of the United States. Luque-Fernandez, Franco, Schomaker, Garitano, D'Este and Williams (2013) are European experts who reside in Spain. They conducted a study of stillbirth events, which intended to analyze the associations of such events with employment status. Their subjects consisted of a multilevel population by observation of births from 2007 to 2010 . Stillbirth was defined as “... the outcome, individual maternal socioeconomic and pregnancy-related characteristics as covariates, and maternal autonomous region of residence as the contextual covariate." To account for differences across regions experts utilized mixed-logic regression models. The totality of the study included 5,560 stillbirth events. In fact, mothers who resided in areas noted for high unemployment were twice as likely to experience a stillbirth event (adjusted OR 2.60; $95 \%$ CI 2.08-3.21). However, this was merely $14 \%$ of an explanation of the problem. For African-born women, i.e.: dark-skinned the chances of a stillbirth event were 1.82 (95\% CI 1.62-2.05) times greater compared to Spanish-born i.e.: light-skinned mothers. In conclusion, dark skin relative to African-born mothers was a significant factor in rates of stillbirth events.

In 1988 experts Alessandri, Stanley, Waddell and Newnham of Australia conducted an investigation pertaining to stillbirth events in Western Australia from 1980-1983. Those mothers who experienced stillbirth events were categorized by demographic correlates including race. Such categories consisted of aboriginal (dark-skinned) and non-aboriginal (light-skinned). Absent race category, the stillbirth rate was determined to be 4.91 per 1,000 total births. Controlling for race via skin color dark-skinned aboriginal mothers were assessed for much higher stillbirth events at 10.80 compared to their light-skinned non-aboriginal counterparts at 4.57 per 1000 total births. Therefore, similar to documented available research dark skin implied by race is a significant factor in stillbirth events according to experts.

\section{Methodology}

The following personal anecdotal evidence pertaining to the implications of dark skin contributed to the methodology for this investigation. Said evidence consisted of secondary qualitative data originally complied by Trivedi (2014).

\section{Stillbirth Clients: A Blog Documentation of Dark Skin}

Client evidence pertaining to skin color relative to stillbirths is readily available as contained in internet blog references by potential clients. Ira Trivedi is a non-Caucasian Mongoloid author who hosts such a blog. Her unedited personal comments addressing skin color is universal in its appreciated denigration of dark skin and offers valuable insight into the implications of skin color for black women confronted with a stillbirth event.

"All my life I have been trained to avoid the sun like the plague. Though I was very sporty as a young girl, I was asked to play indoor sports like badminton, squash and table tennis that would prevent sun exposure. When I late my late teens, I was handed a tube of fairness or whitening cream, which I guilty admit that I still apply - though now there are a hundred more varieties to choose from than the ubiquitous Fair and Lovely whitening cream which I started with and which defined an entire era of Indian 
beauty regimes. In 2010, when I began my research for my upcoming book "India in Love: Marriage and Sexuality in the 21st century," I felt sure that the dark skin stigma would have decreased at least a bit yet I found that - perhaps more than ever before - fair skin defines beauty in this country.

I discovered that skin colour played a major role in the matrimonial market that I had set out to research. I found so many mothers whispering to their sons to take a peek at the feet of prospective brides to detect their true skin colour. After all, makeup, fairness creams or even high-tech whitening lasers are rarely ever applied to the feet! Skin colour played such a major role in determining life-long partnerships that one of the marriage brokers that I met made sure that only bright colour photos were submitted by his clients let there be any confusion about the colour of his or her skin.

The ones who got the brunt of it were the dark skinned girls, no matter how good looking they actually were. I was shocked when an Ivy-league educated doctor, born and brought up in the US, rejected girls solely on complexion, when other factors like education or family background seemed to be a perfect fit. Surprisingly, I found that the obsession with fair skin didn't just stop at men — even women wanted fair husbands, and they weren't shy to ask for it. Mothers of fair daughters chose fair skin over education over weight, and sometimes even over wealth.

Most matrimonial ads today tout skin colour, especially when the person being advertised is fair. Most ads, though, sell skin as the ubiquitous "wheatish," a word describing skin colour that ONLY exists in India, and whose etymology I am still unclear about. That makes me wonder why we don't expand the skin colour palette and base it on other grains-Ricish would be very much in demand, and Jawarish (for Maharashtrians) or Bajarai, for a bit darker than Wheatish but lighter than Jawarish. In Karnataka, there is a grain called Ragi-lighter in colour than wheat, so Karnataka and Tamil Brahmins might well prefer to be referred to as Ragish. And lest we forget Corn, the much-in-demand yellowish northeastern complexion could be Cornish — not to be confused with Cornwall.

But maybe I was just naive to be surprised by the importance that skin colour still holds in our country. After all, just take a look at the Indian consumer market, crowded with umpteen varieties of men's fairness screen, and then the advertising, more robust than ever before, where even men have jumped on the fairness bandwagon, led by the ironically dark-skinned role-model Shahrukh Khan.
Why are we obsessed with skin colour? Some say it's a colonial hang-over, others say it's because of caste, since in the past lower castes worked outdoor in the fields, and were therefore typically dark-skinned. Yet others attribute it to Dravidian-Aryan divide-a defunct debate since recent DNA analysis shows that today north Indians are neither Aryan, nor south Indian Dravidians. We are, in fact, a totally mixed and genetically equal race. It seems that there's no one answer for this national obsession, it's just the way things have always been, and will probably stay for some time to come.

Even I am guilty of propagating the inane concept that fair is beautiful. Every night I slather whitening creams on my face, which are probably bad for me. I buy 100 SPF sunscreens when the melanin in my skin is perfectly capable of keeping me safe in harsh sunlight. Let's hope that the future brings more equality, but for the moment skin is still a major part of the game" (Trivedi, 2014).

By reference to the aforementioned research, expertise and client information evidence dark skin implied by race could be a significant factor in stillbirth events. However, per Eurocentrism as a mainstay of the Social Work academy dark skin does not exist as a standard demographic category but is accessible by application of Evidence-Based Practice (EBP).

\section{The Application of Evidence-based Practice: An Introduction}

Disparities in stillbirth events occur by race worldwide and require resolution. The application of EBP in Social Work has the ability to elevate its practice potential for therapeutic outcomes owed to the medical model of physicians. Some discrepancies exist as pertains to the actual definition of the medical model. Nevertheless, the methodology is a product of the manner in which physicians are trained and how they carry out their work. In conduct of their work, the medical model operated consistently absent challenge and alternative views, which restricted the gathering of information. Subsequently such a method eventually became the unquestioned preference of medical practice. When engaging this practice, in the diagnosis and treatment of disease, physicians then assumed a mechanical view of illness associated with the physicality of the human body (Engel, 1992). Under such circumstances, disease was considered a dysfunction in the operation of the body's routine. Other relevant and perhaps unknown critical aspects of the routine were affectively subjugated or otherwise denied acknowledgement (Biderman \& Herman, 2005). Therefore, the machine-body prevailed as the preferred method and as a functional matter of autonomy. At the onset of illness diagnoses was 
accordingly made relative to the structural or functional failures of the body's physicality. In the aftermath treatment prevailed as a product of standardized diagnostic concepts. These concepts suggest that medical treatment is inclined to be mechanistic and exclusive of such criteria as environmental, psychological and skin color factors. Ultimately, the medical model by such a strict and limited process produced limited potential for resolution of pathologies including stillbirth disparity which necessitated evolutionary change in the form of EBP (Zigmond, 2012).

Similar to the medical model EBP is borrowed by Social Work from the modern-day practice of medicine's evidenced-based approach. One physician named Dr. David Sackett (Sackett, Straus, Richardson, Rosenberg, \& Haynes, 2000) was a major contributor to its origin. The most common definition he proposed pertains to a deliberate and objective use of the currently best available evidence in reaching the most effective, objective and ethical decision-making outcomes as pertains to patient welfare. Doing so may require consultation with leading expertise in the field and research evidence subjected to a systemic and meticulous investigation process (Jarvinen, 2007). Under these EBP circumstances patient and/or client input is a valued commodity as the collection of information is much less restricted. The end result was the triad of EBP information gathering consisting of input from experts, input from clients and access to available documented research. Application of EBP in Social Work practice is heretofore suggested as the most viable practice technology critical to addressing disparities in stillbirth events and other social service challenges.

The aforementioned stillbirth research, the aforementioned references to stillbirth experts and the aforementioned blog text of a potential client pertaining to skin color is application of the EBP triad in the gathering of information evidence. Research, expertise and client blogs are a sensible order of execution for EBP information gathering not necessarily compulsory but effective. The $\mathrm{CDC}$, the WHO and the International Stillbirth Alliance are esteemed bastions of such information evidence. The methodologies of all three research evidence organizations can withstand the most formidable assaults upon the legitimacy of its information product. The international reach of such evidence minimizes the potential for subjectivity and the influences of nationalistic bias. Therefore, upon initial attempts to resolve the disparities of stillbirth events it is scientifically prudent by research evidence to associate skin color with the disparities of such events.

Once skin color is accepted in association with the research on stillbirth events, the gathering of information evidence from noted experts is the second location of the EBP information triad. Based upon their scientific expertise and medical practice skills the works of Hogue and Silver (2011); Luque-Fernandez, Franco, Schomaker,
Garitano, D'Este and Williams (2013); and Alessandri, Stanley, Waddell and Newnham (1988) concur with the available research evidence per associations of skin color with disparities in stillbirth events. The evidence that researchers provide and evidence of the $\mathrm{CDC}$, the WHO and the International Stillbirth Alliance, are not limited to any one geographical location or racial group. Experts who concur with available research are similarly diverse in the processing of their information evidence, which establishes the rudiments of a pattern. Said pattern is then reason for additional confidence in the association of skin color with disparities in stillbirth events that can be utilized for resolution or reaching a preferred therapeutic outcome. In the aftermath is left the query of skin color specificity answered as dark skin concerning what manner of color provokes stillbirth disparity.

Client input is the third information triad of EBP information gathering. Information gathering using the medical model would not accommodate such an input. The databases of the $\mathrm{CDC}$, the $\mathrm{WHO}$ and the International Stillbirth Alliance are not constructed in such a way as to access skin color information. Experts similarly directed by race-based Eurocentric standards likewise omit skin color from the details of their information evidence (Kumar, 2015). EBP in its acknowledgement of client information evidence incorporates skin color details when mentioned otherwise inaccessible. Therefore, application of the EBP model allows for critical client information that may be considered medical model insignificant. The more relevancy of skin color established by research and expert contribution as implied by race is specified as dark skin by the more encompassing client input as in the previous internet blog. This type of additional client input characteristically remains at the forefront of social ills including stillbirth events. Unfortunately, the Eurocentric standards of both medicine and Social Work practice are not equipped to assess the implications of skin color for stillbirth disparities absent EBP (Robinson-Moore, 2008). While research and expert input acknowledges skin color only by client participation are the details subject to explanation in relation to dark skin specifically.

As per client information evidence, having dark skin by EBP methods is assessed as a contributing factor in stillbirth disparities. For black women in particular, dark skin is an impediment to their having internalized an alien beauty ideal (Karupiah, 2015). Therefore, in an effort to acquire the beauty ideal of light skin they apply bleaching creams to their skin as suggested by client blog evidence. Such creams may contain arsenic, mercury and other toxic substances that can reach the fetus via the placenta resulting in stillbirth events (Ben, Alexis, Mohamed, Wang, Khan, et. al., 2016). Caucasian and Mongoloid race mothers have less need for bleaching creams due to their genetic proximity to the light skin ideal. Subsequently via application of the EBP model to the gathering of information evidence, stillbirth disparities via dark skin is 
suggested as a viable, more prudent explanation of the disparity problem. Once the problem can be adequately explained, it becomes amenable to scientific application where stillbirth disparities may diminish or be eliminated entirely. Thus, by EBP Social Work practice is poised to make a major contribution to the resolution of a social ill. If by EBP dark skin is stillbirth critical and if black women utilize toxic bleaching creams to lighten their dark skin, Social Work practitioners advising such women to withhold the use of these creams when they become pregnant will eliminate exposure of the fetus to bleaching cream toxins. In the aftermath, stillbirth disparities will likely be reduced in proportion to the abolition of an unnecessary toxic risk.

\section{Discussion, Implications and Conclusions}

The information triad of EBP is ultimately gleaned from the practitioner's accumulated experience, their education and their practice skills. In a values-profession such as Social Work, the client is equally appreciated for their unique perspective, personal experiences, individual concerns, expectations, and social system functions. Their contributions facilitate the ultimate in research evidence because the application of EBP emphasizes sound data collection methodologies (Thorsteinsson \& Sveinsdottir, 2014). Subsequently absent EBP and given to the limitations of traditional evidence collection procedures practitioners cannot suffice in a prudent decision-making manner. The serious consideration of client input, practitioner expertise and the quality of scientific evidence will ultimately serve in the best interest of both client well-being and the goals of the Social Work profession at-large (Weiss, 2003). In this manner, the client served by a Social Work practitioner will have the greatest access to preferred quality of life and desired social service outcomes.

The initiation of EBP is normally instigated by some client action or client status, which requires professional attention. By client input are generated queries in the assessment of therapy, the effectiveness of diagnostic attention, the forecast of pathology or the looming potential of a disorder. In Social Work practice EBP may be put to use as a tool for the investigation of problem-solving strategies whereby progress can be actively sustained (Artistico, Cervone \& Pezzuti, 2003). Measuring client outcomes statistically by EBP application is a critical aspect of this process that will allow for the replacement of antiquated, ineffective methods when necessary. Otherwise, the subjugation of statistics by less professional measures including tradition, gut reaction, single observations or unsubstantiated acts of habit will prevail without question.

In the EBP meticulous collection of data, expert consultation and client input the resolution of stillbirth disparities will be less subject to inferior problem-solving strategies (Mosconi, 2003). Therefore, improvement of the current circumstances of stillbirth events is the necessary replacement of race with the more measurable dimensions of skin color. Initially this will present a formidable challenge and hence threat to the Eurocentric status quo. That's because the history of the Social Work academy has been the Eurocentric manufacture of information, not necessarily intended but a factor nonetheless (Soydan, 2012). That history then defines all existential matters in proximity to Eurocentric experience, Eurocentric norms and as pertains to race versus skin color Eurocentric preferences. Subsequently in traditions of the Social Work academy and its dedication to the demographic category of race is omission of arguably the most critical factor associated with stillbirth events and other pathologies attributable to dark skin. That omission has sustained disparities in stillbirth events and a host of problems otherwise unnecessary. Alternatively, the ultimate application of EBP has the potential to illuminate and eventually resolve these shortcomings in the most effective and least polarizing manner.

Despite its relative omission, there has been a recent accumulation of research pertaining to dark skin not irrelevant as a factor in stillbirth events (Dodds, King, Armson, Allen, et. al., 2006). Unfortunately, reference to skin color has been denigrated as exotic or irrelevant for mainstream purposes. Upon closer observation, it is dramatically revealed that skin color has potential application to any number of social issues equal to and perhaps exceeding that of race. The measurability of skin color and its biology-based definition serves scientific investigation that would render all manner of race category obsolete. Therefore, EBP as applied in Social Work has the potential to advance the profession thereby sustaining its prestige, which is critical for a profession where public sanction is a basic requirement for practice (Biggerstaff, 2000). Furthermore, EBP is also much less challenging to replicate for future or alternative studies introduced into the public domain and thereby further educating a need-to-be informed public.

Lastly, human nature has historically subscribed to the notion of group category. Race, religion, culture, etc. have all served to denote an in-group out-group mentality. Subsequently maximum research integrity in this a heterogeneous era becomes virtually impossible by such an arrangement. In the outcome, access to an unadulterated knowledge base is disserved. Such access is exceedingly compulsory in advanced Western societies where human diseases evolve commensurate with advances in social service technology (Youn, 2007). Application of skin color in the study of stillbirth disparities can greatly reduce events among dark-skinned mothers. Absent EBP, such events cannot be addressed in dark skin because dark skin is not validated by a Eurocentric epistemology. Alternatively, skin color via EBP incorporates the notion of humanity exceeding all racial, religious, and geographic 
preferences. Furthermore, the mathematical measure of skin color given to the scientific method fosters objectivity and is much more conducive to civil discourse and hence problem-solving resolution (Lynch, 2005). By eliminating race from the dynamics of scientific investigation via EBP Social Work will be served in meeting its challenges well into the $21^{\text {st }}$ century and beyond.

\section{REFERENCES}

Alessandri, L., Stanley, F., Waddell, V. \& Newnham, J. (1988). Stillbirths in Western Australia 1980-1983: Influence of race, residence and place of birth. Australian and New Zealand Journal of Obstetrics, 28(4), 284-292.

Aristico, D., Cervone, D. \& Pezzuti, L. (2003). Perceived self-efficacy and everyday problem solving among young and older adults. Psychology and Aging, 18(1), 68-79.

Benn, E., Alexis, A., Mohamed, N., Wang, Y., Khan, I., et. al. (2016). Skin bleaching and dermatologic health of African and Afro-Caribbean populations in the US: New directions for methodologically rigorous, multidisciplinary, and culturally sensitive research. Dermatology and Therapy, 6(4), 453-459.

Biderman, A., Yeheskel, A. \& Herman, J. (2005). The biopsychosocial model - have we made any progress since 1977 ? Family Systems \& Health, 23(4), 379-386.

Biggerstaff, M. (2000). Development and validation of the social work career influence questionnaire. Research on Social Work Practice, 10(1), 34-54.

CDC. (2015). Stillbirth (fetal death) rates, by race and Hispanic origin of mother: United States, 2013. Retrieved on 6/27/17 from https://www.cdc.gov/ncbddd/stillbirth/data-stillbirth-race-text.ht $\mathrm{ml}$.

Dodds, L., King, W., Fell, D., Armson, B., Allen, A., et. al. (2006) Stillbirth risk factors according to timing of exposure. Annals of Epidemiology, 16(8), 607-613.

Dubriwny, T., Bates, B. \& Bevan, J. (2004). Lay understandings of race: Cultural and genetic definitions. Community Genetics, 7(4), 185-195.

Engel, G. (1992). The need for a new medical model: A challenge for biomedicine. Family Systems Medicine, 10(3), 317-331.

Garn, S. \& Coon, C. (2009). On the number of races of mankind. American Anthropologist, 57(5), 996-1001.

Gibbs, R. (2006). Q can stillbirth be predicted? OBG Management, 18(5), 14-18.

Glasker, W. (2014). Color matters: skin tone bias and the myth of post racial America. Middletown, 52(1), 143.

Hogue, C. \& Silver, R. (2011). Racial and ethnic disparities in United States: Stillbirth rates: Trends, risk factors, and research needs. Seminars in Perinatology, 35(4), 221-233.

Hu, D. (2008). Methodology for evaluation of melanin content and production of pigment cells in vitro
[dagger]Photochemistry and Photobiology, 84(3), 645-649.

International Stillbirth Alliance (2009). The Partnership for Maternal, Newborn and Child Health. Retrieved on 5/22/17 from http://www.who.int/pmnch/media/news/2011/stillbirths_country rates.pdf

Jarvinen, P. (2007). Action research is similar to design science. Quality and Quantity, 41(1), 37-54.

Karupiah, P. (2015). Have beauty ideas evolved? Reading and beauty ideas in Tamil movies by Malaysian Indian youths Sociological Inquiry, 85(2), 239-261.

King, G., Yerger, V., Whembolua, G., Bendel, R., Kittles, R. \& et.al. (2009). Link between facultative melanin and tobacco use among African Americans. Pharmacology Biochemistry and Behavior, 92(4), 589-586.

Krause, M. (2012). Measurement validity is fundamentally a matter of definition, not correlation. Review of General Psychology, 16(4), 391-400.

Kumar, S. \& Amin, S. (2015). Eurocentrism: modernity, religion and democracy: a critique of Eurocentrism and culturalism. Journal of Intercultural Studies, 36(1), 106-108.

Luque-Fernandez, M., Franco, M., Gelaye, B., et al. (2013). Unemployment and stillbirth risk among foreign-born and Spanish pregnant women in Spain, 2007-2010: a multilevel analysis study. European Journal of Epidemiology, 28(12), 991-999.

Lynch, W. (2005). The ghost of Wittgenstein: Forms of life, scientific method, and cultural critique. Philosophy of the Social Sciences, 35(2), 139-174.

Mosconi, G. (2003). Research on "problem solving." Sistemi Intelligenti, 15(1), 103-105.

Murdoch, B. (2009). Editorial. Asia Pacific Journal of Speech, Language and Hearing, 12(1), ix.

Ore, T. (2011). The Social Construction of Difference and Inequality: Race, Class, Gender and Sexuality. Dubuque: McGraw-Hill.

Robinson-Moore, C. (2008). Beauty standards reflect Eurocentric paradigms-So what? Skin color, identity and Black female beauty. The Journal of Race and Policy, 4(1), 66-85.

Sackett, D. L.; Straus, S. E.; Richardson, W. C.; Rosenberg, W.; \& Haynes, R. M. (2000). Evidence-based medicine: How to practice and teach EBM (2nd Ed.). New York: Churchill Livingstone.

Soh, M., Nelson-Piercy, Dib, F., Westgren, M., McCowan, L., et al. (2015). Brief Report: Between pregnancy outcomes and death from cardiovascular causes in parous women with systemic lupus erythematosus: A study using Swedish population registries. Arthritis \& Rheumatology, 67(9), 2376-2382.

Soydan, H. (2012). Understanding social work in the history of ideas. Research on Social Work Practice, 22(5), 468-480.

Thorsteinsson, H. \& Sveinsdottir, H. (2014). Readiness for and predictors of evidence-based practice of acute care nurses: A cross sectional postal survey. Journal of Caring Sciences, 28(3), $572-581$. 
Trivedi, I. (2014). The color of my skin. Retrieved on $6 / 27 / 17$ from

http://blogs.timesofindia.indiatimes.com/india-in-love/the-colour -of-my-skin/.

Tyler, C., Grady, S., Grigorescu, V., Luke, B., Todem, D., et al. (2012). Impact of fetal death reporting requirements on early neonatal and fetal mortality rates and racial disparities. Public Health Reports, 127(5), 507.

Weiss, I. (2003). Social work students and social change: on the link between views of poverty, social work goals and policy practice. International Journal of Social Welfare, 12(2), 132-141.

Youn, E. (2007). The relationship between technology content in a masters of social work curriculum and technology use in social work practice: A qualitative research study. Journal of Technology in Human Services, 25(1-2), 45-58.

Zigmond, D. (2012). The medical model-its limitations and alternatives. Retrieved on 6/27/17 from

$\mathrm{http}: / /$ marco-learningsystems.com/pages/david-zigmond/medical -model.htm. 